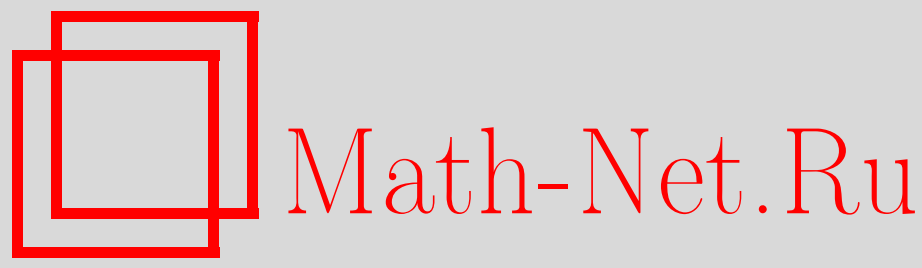

М. Гнатич, М. В. Комарова, М. Ю. Налимов, Микроскопическое обоснование стохастической F-модели критической динамики, ТМФ, 2013, том 175, номер 3, 398-407

DOI: https://doi.org/10.4213/tmf8482

Использование Общероссийского математического портала Math-Net.Ru подразумевает, что вы прочитали и согласны с пользовательским соглашением http://www.mathnet.ru/rus/agreement

Параметры загрузки:

IP : 18.207 .199 .55

26 апреля 2023 г., 07:16:54

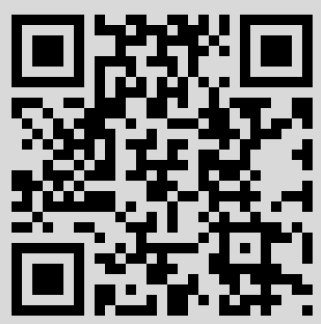




\title{
МИКРОСКОПИЧЕСКОЕ ОБОСНОВАНИЕ СТОХАСТИЧЕСКОЙ Г-МОДЕЛИ КРИТИЧЕСКОЙ ДИНАМИКИ
}

\begin{abstract}
На основе микроскопического подхода выведены стохастические уравнения Ланжевена квантовой бозе-жидкости в окрестности фазового перехода в сверхтекучее состояние. Локальная форма уравнений получается при учете конечности размеров системы либо ограниченности рассматриваемых времен. Предъявлены микроскопические выражения для случайных сил стохастических уравнений. Показано, что приближение белого шума справедливо лишь в критической области. Доказано, что адекватной моделью описания критических флуктуаций является именно F-модель.
\end{abstract}

Ключевые слова: стохастические динамические модели, уравнение Ланжевена, F-модель, критическая динамика, бозе-жидкость, сверхтекучесть, временнь́е функции Грина.

DOI: $10.4213 / \operatorname{tmf} 8482$

\section{1. ВВЕДЕНИЕ}

Настоящая работа посвящена описанию динамики квантовой бозе-жидкости в окрестности сверхтекучего фазового перехода. Известно, что данную систему описывает стохастическая F-модель в стандартной классификации [1], которая в окрестности фазового перехода сводится [2], [3] к Е-модели в той же классификации. В работе [4] была предпринята попытка включения в рассмотрение для данных моделей гидродинамических мод. При этом в результате анализа ренормгрупповых уравнений оказалось, в частности, что критическая размерность вязкости, определяющая динамику равновесной системы в окрестности сверхтекучего фазового перехода, существенно зависит от того, какая именно стохастическая модель рассматривается, Е-модель или F-модель.

Кроме того, в работах [2], [3] из феноменологических соображений утверждалось, что F-модель более представительна и поэтому более пригодна для описания

*Institute of Experimental Physics, Slovak Academy of Sciences, Košice, Slovakia. E-mail: hnatic@saske.sk

${ }^{\dagger}$ Санкт-Петербургский государственный университет, Санкт-Петербург, Россия. E-mail: frommarina@mail.ru, myunalimov@gmail.com 
рассматриваемого фазового перехода. Однако в свете поставленной задачи данное утверждение требует более строгого с микроскопической точки зрения обоснования. Попытка такого обоснования и предпринята в настоящей статье.

Наш анализ основан на модели, которую мы считаем возможным называть микроскопической - базирующейся на модели квантового бозе-газа с взаимодействием типа плотность-плотность. Данная модель является достаточно известной, она используется в классических монографиях для описания фазовых переходов в квантовых системах [5]. Следуя традиции, мы рассматриваем равновесные флуктуации бозе-системы в большом каноническом ансамбле при конечной температуре, используя химический потенциал в качестве "управляющего параметра".

В настоящей работе мы использовали приближение локального взаимодействия стандартное при описании критического поведения в квантовых моделях [5]. Впрочем, как это следует из наших результатов, нелокальность взаимодействия не повлияла бы на описываемый метод.

Целью работы было получить из микроскопических принципов стохастические уравнения Ланжевена описываемой системы. Для этого использовалась та же идея, что и при выводе уравнения броуновского движения, когда мелкомасштабное хаотическое движение рассматривается в качестве случайной силы - белого шума, а уравнения движения записываются для крупномасштабных движений броуновских частиц, находящихся в поле этого шума.

Интересуясь динамикой равновесных флуктуаций, мы начали с формализма временны́х функций Грина при конечной температуре, в значительной степени забытого в последнее время. При этом, для экономии объема, мы не рассматривали вопрос о контурах интегрирования по комплексному параметру "времени” и об аналитических свойствах временнь́х функций Грина при конечной температуре, позволяющих деформировать данный контур. Заинтересованный читатель может найти обсуждение данных вопросов в классических трудах (см., например, [6]). В разделе 2 записаны уравнения Швингера, в разделе 3 мы вывели нелокальные уравнения для поля параметра порядка, обратившись при этом к формализму функциональных преобразований Лежандра [7]. Введя в разделе 4 в рассмотрение поле плотности, мы построили в разделе 5 теорию возмущений, которая свела уравнения в критической области к локальной форме. В разделе 6 показано, что отдельные члены уравнений фактически играют роль случайной силы, что превращает наши уравнения в стохастические. В разделе 7 мы показали соответствие полученных уравнений и стохастических уравнений F-модели критической динамики.

\section{2. УРАВНЕНИЯ ШВИНГЕРА}

Рассмотрим квантово-полевую модель комплексного поля $\psi(\mathbf{x}, t), \psi(\mathbf{x}, t)^{+}, \mathbf{x} \in \mathbb{R}$, с действием

$$
S\left(\psi, \psi^{+}\right)=\psi^{+}\left(\partial_{t}-\frac{\Delta}{2 m}-\mu\right) \psi+\frac{\lambda}{2}\left(\psi^{+} \psi\right)^{2},
$$

где $\Delta$ - оператор Лапласа в пространстве $\mathbb{R}, \mu$ - химический потенциал, $\lambda$ - константа связи, интегрирование по аргументам $\mathbf{x}$ и $t$ подразумевается. В формализме температурных функций Грина параметр $t$ является комплексным, его вещественная часть соответствует обычному времени $\tau$, а мнимая - обратной температуре, $\operatorname{Re} t \in(0, \tau), \operatorname{Im} t=\beta \in(0,1 / k T)$. 
Введем в импульсном пространстве параметр $\Lambda$, условно отделяющий мелкомасштабные явления от крупномасштабных. Поле $\psi$ можно представить в виде суммы мягкого поля $\varphi$ и жесткого поля $\xi$, каждое из которых соответствует либо только крупномасштабным, либо только мелкомасштабным явлениям: при $k<\Lambda$ поле $\xi(k)$ обращается в нуль, $\psi(k)=\varphi(k)$; при $k>\Lambda$ исчезает поле $\varphi(k)$, и $\psi(k)=\xi(k)$. Фактически это замена переменных в функциональном интеграле:

$$
\int \mathcal{D} \psi \mathcal{D} \psi^{+} e^{-S\left(\psi, \psi^{+}\right)} \underset{\psi \equiv \varphi+\xi}{\longrightarrow} \int \mathcal{D} \varphi \mathcal{D} \varphi^{+} \mathcal{D} \xi \mathcal{D} \xi^{+} e^{-S\left(\varphi, \varphi^{+}, \xi, \xi^{+}\right)} .
$$

В качестве параметра порядка в Е- и F-моделях критической динамики рассматриваются поля, усредненные по ансамблю. Чтобы описать эволюцию таких средних полей, рассмотрим уравнения Швингера для действия (1) с источником внешнего поля $A$

$$
\int \mathcal{D} \varphi \frac{\delta}{\delta \varphi} e^{-S+A^{+} \varphi+A \varphi^{+}}=\left\langle-\frac{\delta S}{\delta \varphi}+A^{+}\right\rangle=0
$$

где введено обозначение $\langle\cdot\rangle$ для усреднения с весом $e^{-S}$ (усреднение, как и интегрирование в (2), проводится только по мягким полям). При нулевых значениях источника, т. е. в отсутствие внешнего поля, это дает

$$
L\left\langle\varphi^{+}\right\rangle+\lambda\left\langle\psi^{+} \psi \psi^{+}\right\rangle=0, \quad L \equiv-\partial_{t}-\frac{\Delta}{2 m}-\mu .
$$

Второе уравнение Швингера получается из (2) заменой $\varphi$ на $\varphi^{+}$, что дает комплексно-сопряженное уравнение, в котором $L^{*}=\partial_{t}-\Delta / 2 m-\mu$. Эти уравнения можно переписать в импульсно-частотном представлении; при малых значениях внешнего импульса $\mathbf{k}$ это приводит уравнение (3) к виду

$$
L\left\langle\varphi^{+}(\omega, \mathbf{k})\right\rangle+\lambda\left\langle\varphi^{+} \varphi \varphi^{+}\right\rangle+2 \lambda\left\langle\varphi^{+}\right\rangle \xi \xi^{+}+\lambda\langle\varphi\rangle \xi^{+} \xi^{+}+\lambda \xi^{+} \xi \xi^{+}=0 .
$$

Оператор $L$ в данном представлении, очевидно, соответствует выражению $L(\omega, \mathbf{k})=$ $\left(i \omega+\mathbf{k}^{2} / 2 m-\mu\right)$, а кубические по полям вклады подразумеваются в виде сверток. Слагаемые $\varphi \varphi \xi$ с различными расстановками знаков сопряжения здесь отброшены по закону сохранения импульса: соответствующие члены в импульсном представлении имеют вид свертки $\int d \mathbf{k}_{1} d \mathbf{k}_{2} d \mathbf{k}_{3} \varphi\left(\mathbf{k}_{1}\right) \varphi\left(\mathbf{k}_{2}\right) \xi\left(\mathbf{k}_{3}\right)$, где область интегрирования $\mathbf{k}_{1}+\mathbf{k}_{2}+\mathbf{k}_{3}=\mathbf{k}$ исчезает при малых $\mathbf{k}_{1}, \mathbf{k}_{2}, \mathbf{k}$ и больших $\mathbf{k}_{3}$. Соответствующая процедура является классической и обоснована в работах, посвященных вильсоновской ренормгруппе (см. [8]).

Отождествим усредненные по ансамблю мягкие поля $\langle\varphi(\mathbf{k})\rangle$ и $\left\langle\varphi^{+}(\mathbf{k})\right\rangle$ с параметром порядка системы. Наша цель - показать, что они подчиняются стохастическим уравнениям Ланжевена, соответствующим F-модели критической динамики. Вклад $\xi^{+} \xi \xi^{+}$в дальнейшем будет отождествлен со случайной аддитивной силой, порождаемой мелкомасштабными явлениями, в поле которой находится макросистема. Технически это окажется возможно сделать при помощи математического аппарата функционального преобразования Лежандра, хорошо приспособленного для описания систем в поле внешней силы [7]. 


\section{3. ПРЕОБРАЗОВАНИЕ ЛЕЖАНДРА}

Рассмотрим производящий функционал связных функций Грина

$$
W(A)=\ln \left\langle e^{\varphi^{+} A+\varphi A^{+}}\right\rangle,
$$

который в результате $n$-кратного варьирования по полям из набора $A, A^{+}$порождает набор функций $W_{n}$. Чтобы различать при необходимости разные функции порядка $n$, будем перечислять поля, по которым варьировался производящий функционал, например

$$
W_{A A A^{+}} \equiv \frac{\delta^{3} W}{\delta A \delta A \delta A^{+}} \in W_{3},\left.\quad W_{A A A^{+}}\right|_{A, A^{+}=0}=\left\langle\varphi^{+} \varphi^{+} \varphi\right\rangle .
$$

Введем компактную запись для параметра порядка - среднего поля $\langle\varphi\rangle$ :

$$
\alpha \equiv\langle\varphi\rangle=\left.W_{A^{+}}\right|_{A, A^{+}=0}, \alpha^{+} \equiv\left\langle\varphi^{+}\right\rangle=\left.W_{A}\right|_{A, A^{+}=0} .
$$

Функционал, полученный при помощи преобразования Лежандра

$$
\Gamma(\alpha)=W\left(A, A^{+}\right)-\alpha A^{+}-\alpha^{+} A,
$$

и функции $\Gamma_{n}$, полученные $n$-кратным варьированием его по полям $\alpha, \alpha^{+}$, связаны с исходными переменными соотношениями $\Gamma_{\alpha}=-A^{+}, \Gamma_{\alpha^{+}}=-A, W_{2}=$ $\left(-\Gamma_{2}\right)^{-1}$. Последнее равенство следует воспринимать как матричное, подразумевая, что компоненты $(2 \times 2)$-матрицы $W_{2}$ получаются варьированием пары функционалов $\left\{W_{A^{+}}, W_{A}\right\}$ по паре полей $\left\{A^{+}, A\right\}$, а компоненты $\Gamma_{2}$ - варьированием $\left\{\Gamma_{\alpha}, \Gamma_{\alpha^{+}}\right\}$ по паре $\left\{\alpha, \alpha^{+}\right\}$[7]. Отметим, что $W_{2}$ совпадает с матрицей связного полного пропагатора.

Новые переменные приводят уравнения Швингера к форме (ср. с (2))

$$
\left\langle\frac{\delta S}{\delta \varphi}\right\rangle=-\Gamma_{\alpha}, \quad\left\langle\frac{\delta S}{\delta \varphi^{+}}\right\rangle=-\Gamma_{\alpha^{+}},
$$

при нулевых значениях источников они принимают простой вид $-\Gamma_{\alpha}=0,-\Gamma_{\alpha^{+}}=0$. Это позволяет получать матрицу $-\Gamma_{2}$ варьированием левой части уравнений Швингера (4) по полям $\alpha, \alpha^{+}$.

Уравнения Швингера в терминах полей $\alpha, \alpha^{+}$дают $-\Gamma_{\alpha}=0$, или

$$
L \alpha^{+}+\lambda\left(2 \alpha^{+} \xi \xi^{+}+\alpha \xi^{+} \xi^{+}+\alpha \alpha^{+} \alpha^{+}+\xi \xi^{+} \xi^{+}+2 \mathcal{K}_{1} \alpha^{+}+\mathcal{K}_{2} \alpha+\mathcal{L}\right)=0,
$$

функции $\mathcal{K}_{1}, \mathcal{K}_{2}$ и $\mathcal{L}$ задаются диаграммами

$$
\mathcal{K}_{1}=\Omega, \quad \mathcal{K}_{2}=\Omega, \quad \mathcal{L}=
$$

с пропагаторами, определяемыми матрицей $W_{2}$ :

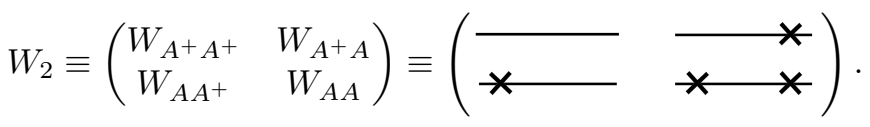


В (6) и ниже точки показывают внешние аргументы диаграмм в петлевых выражениях для $\mathcal{L}$ треххвостым объектом $\Gamma_{3}$ обозначена сумма элементов множества $\Gamma_{3}$ со всевозможной расстановкой пары полей $\left\{\alpha, \alpha^{+}\right\}$на хвостах, свернутых с пропагаторами. Комплексно-сопряженное уравнение $-\Gamma_{\alpha^{+}}=0$ пишется аналогично.

В дальнейшем нам придется обсуждать уравнение (5) и подобные ему как в координатно-временном, так и в импульсно-частотном представлениях. При этом кубические по полям слагаемые и вклады типа $\mathcal{K}_{1} \alpha^{+}$в фурье-пространстве понимаются в виде сверток, а в координатном пространстве - в виде произведений функций с совпадающими аргументами.

Варьируя уравнение (5) по паре полей $\left\{\alpha, \alpha^{+}\right\}$и учитывая, что пропагаторы в диаграммных слагаемых также зависят от полей, получим матрицу обратных пропагаторов в виде

$$
\begin{aligned}
& -\Gamma_{\alpha \alpha}=\lambda\left(\alpha^{+} \alpha^{+}+\xi^{+} \xi^{+}+\mathcal{K}_{(++)}+\right. \\
& +2 \alpha+\Gamma_{3}+\alpha+\Gamma_{3}+\Gamma_{4}^{*}+\Gamma_{3}+ \\
& -\Gamma_{\alpha^{+} \alpha}=L(\omega, \mathbf{k})+\lambda\left(2 \alpha \alpha^{+}+2 \xi \xi^{+}+2 \mathcal{K}_{(+)}+\right. \\
& \left.+2 \alpha+{ }^{*}+\alpha+\Gamma_{3}^{*}+\Gamma^{*}+\Gamma_{3}^{*}+\Gamma_{3}\right) .
\end{aligned}
$$

В петлевых выражениях с $\Gamma_{3}$ следует суммировать лишь те элементы множества $\Gamma_{3}$, которые соответствуют различной расстановке крестов на свернутых хвостах; расстановка крестов на внешних хвостах элементов $\Gamma_{3}$ указана явно крестами над символами • внешних хвостов. Заметим, что ни здесь, ни далее нам не потребовалось обсуждать такие свойства наших диаграмм, как связность и 1-неприводимость.

\section{4. ПОЛЕ ПЛОТНОСТИ}

Объектом исследования обсуждаемой модели, кроме полей $\alpha, \alpha^{+}, \xi, \xi^{+}$, является поле плотности системы, усредненное по $\alpha, \alpha^{+}$:

$$
M \equiv\left\langle\psi^{+} \psi\right\rangle=\left\langle\left(\varphi^{+}+\xi^{+}\right)(\varphi+\xi)\right\rangle=\left\langle\varphi^{+} \varphi\right\rangle+\left\langle\xi^{+} \xi\right\rangle
$$

Здесь члены $\left\langle\varphi^{+} \xi+\xi^{+} \varphi\right\rangle$ отброшены, как и в разделе 2 , по закону сохранения импульса. Из равенства $\left\langle\varphi^{+} \varphi\right\rangle=\alpha^{+} \alpha+\mathcal{K}_{1}$, следующего из явного вида преобразования Лежандра, легко видеть, что

$$
M \equiv \alpha^{+} \alpha+\xi^{+} \xi+\mathcal{K}_{1}
$$

Кроме того, поле $M$ явно выделяется в первой строке выражения (8), т. е. в однопетлевом приближении $-\Gamma_{\alpha^{+} \alpha}=L+2 \lambda M$. 
Введя в рассмотрение новое поле, мы должны ввести и уравнение, задающее его динамику. Это легко сделать, рассмотрев по аналогии с (2) выражения

$$
I_{1}=\int \mathcal{D} \psi \mathcal{D} \psi^{+} \psi \frac{\delta e^{-S}}{\delta \psi}, \quad I_{2}=\int \mathcal{D} \psi \mathcal{D} \psi^{+} \psi^{+} \frac{\delta e^{-S}}{\delta \psi^{+}} .
$$

Перебросим в каждом из них вариационную производную под знаком интеграла и получим тождественные выражения, т. е. убеждаемся, что $I_{1}=I_{2}$. Вычитая одно из другого и подставляя явный вид $S$, получим

$$
\partial_{t} M=\left\langle\psi^{+} \partial_{t} \psi+\psi \partial_{t} \psi^{+}\right\rangle=\left\langle\psi \frac{\Delta}{2 m} \psi^{+}\right\rangle-\left\langle\psi^{+} \frac{\Delta}{2 m} \psi\right\rangle,
$$

что после перехода к переменным $\alpha, \alpha^{+}$дает требуемое динамическое уравнение для поля плотности

$$
2 m \partial_{t} M=\alpha^{+} \Delta \alpha-\alpha \Delta \alpha^{+}+\Delta_{-} \Omega_{+}\left(\xi^{+} \Delta \xi-\xi \Delta \xi^{+}\right) .
$$

Петлевые вклады здесь содержат в координатном представлении оператор Лапласа, действующий на один из концов пропагатора, что изображено символами $\Delta$, расположенными на линиях в диаграммах.

\section{5. ТЕОРИЯ ВОЗМУЩЕНИЙ}

В разделе 3 мы получили комплексно-сопряженные динамические уравнения для полей $\alpha$ и $\alpha^{+}(5)$. Уравнения оказались нелокальными, соответствующие нелокальные вклады представлялись диаграммами типа $\mathcal{K}$ и $\mathcal{L}$; обратная матрица пропагаторов (7), (8) получалась варьированием этих уравнений и также содержала петли. Тем не менее для системы можно построить теорию возмущений по числу петель, если ограничиться рассмотрением инфракрасной (ИК) области, т. е. окрестностью точки фазового перехода.

Действительно, будем строить ряды теории возмущений по полям, полагая в каждом порядке теории возмущений малыми также внешнюю частоту и импульс в диаграммах и разлагая по ним в ряды. При этом мы предполагаем аналитичность данных рядов, поскольку рассматриваем систему конечного размера.

Начиная с беспетлевого приближения для $\Gamma_{2}($ ср. с (7), (8))

$$
-\Gamma_{\alpha \alpha}=\lambda\left(\alpha^{+} \alpha^{+}+\xi^{+} \xi^{+}\right), \quad-\Gamma_{\alpha^{+\alpha}}=L(\omega, \mathbf{k})+\lambda\left(2 \alpha \alpha^{+}+2 \xi \xi^{+}\right),
$$

обладающего, очевидно, локальностью, вычислим пропагаторы, а также $\Gamma_{3}, \Gamma_{4}$, ответ представим в виде рядов по полям $\alpha, \alpha^{+}, \xi, \xi^{+}$. Несложно видеть, что ряды для пропагаторов $W_{A A}, W_{A^{+} A^{+}}$начинаются с квадратичного по полям члена, в то время как перекрестные пропагаторы $W_{A A^{+}}, W_{A^{+} A}$ благодаря члену $L(\omega, \mathbf{k})$ в $\Gamma_{\alpha+\alpha}$ конечны при малых полях. После подстановки полученных пропагаторов в $(5)$ (в диаграммные вклады $\mathcal{K}, \mathcal{L}$ ) следует разложить диаграммы в ряды по внешним импульсам $\mathbf{k}$ и частотам $\omega$. При этом здесь (как и в последующих порядках теории возмущений) следует удерживать только вклады, либо квадратичные по $\mathbf{k}$, либо линейные по частоте, либо квадратичные по полям. Остальные слагаемые 
данных уравнений являются ИК-несущественными; подтверждающий это размерный счет полностью повторяет известный размерный анализ F-модели критической динамики [7]. В результате в первом порядке теории возмущений динамическое уравнение (5) дает

$$
\left(c_{1} i \omega+\frac{c_{2} \mathbf{k}^{2}}{2 m}-c_{3} \mu\right) \alpha^{+}+\lambda\left(2 c_{4} \alpha^{+} \xi \xi^{+}+c_{5} \alpha \xi^{+} \xi^{+}+c_{6} \alpha \alpha^{+} \alpha^{+}+c_{7} \xi^{+} \xi \xi^{+}\right)=0
$$

где числовые множители $c_{i}$ появляются в результате разложения диаграмм $\mathcal{L}, \mathcal{K}$ в ИК-области.

Итак, мы видим, что нелокальные вклады диаграмм фактически просто перенормировали локальные слагаемые уравнения (5). Например, вклад $\mathcal{K}_{1} \alpha^{+}$в (5) приводит лишь к перенормировке слагаемых $\mu \alpha^{+}, \alpha \alpha^{+} \alpha^{+}$и $\alpha^{+} \xi \xi^{+}$; вклад $\mathcal{K}_{2} \alpha$ дает перенормировку только $\alpha \alpha^{+} \alpha^{+}$и $\alpha \xi^{+} \xi^{+}$, поскольку соответствующий этой петле пропагатор $W_{A A}$ мал (квадратичен по полям). Аналогично можно видеть, что член $\mathcal{L}$ перенормирует все локальные члены.

Варьируя полученные локальные динамические уравнения предыдущей итерации, мы получим следующее приближение для пропагаторов, которое снова будет локальным. Повторение описанной процедуры приводит к тому, что на каждом шаге теории возмущений мы получаем исправленную форму динамических уравнений, отличающуюся от уравнений предыдущей итерации лишь исправленными множителями $c_{i}$.

Преобразуясь в ходе каждого шага теории возмущений, коэффициенты $c_{i}$ в результате заданного (большого) числа итераций превратятся в числовые коэффициенты, которые мы обозначим $\widetilde{C}_{i}$.

Полученные уравнения удобно переписать, используя введенное ранее поле плотности (9), - это позволит нам получить микроскопические аналоги феноменологических величин F-модели критической динамики, которая традиционно формулируется в терминах полей $\alpha, \alpha^{+}, M[7]$. Для этого достаточно перегруппировать вклады с коэффициентами $\widetilde{C}_{3}, \widetilde{C}_{4}, \widetilde{C}_{6}$, т. е. записать их в виде

$$
\alpha^{+}\left(-\widetilde{C}_{3} \mu+2 \lambda \widetilde{C}_{4} \xi \xi^{+}+\widetilde{C}_{6} \lambda \alpha \alpha^{+}\right)=\alpha^{+}\left(C_{0} M+C_{3}+C_{4} \xi \xi^{+}+C_{6} \alpha \alpha^{+}\right) .
$$

Разумеется, при определении $C_{0}$ имеется произвол, который, как будет видно ниже, не влияет на критическое поведение системы. Пока же положим лишь $C_{0} \neq 0$, чтобы не исключать поле $M$ из рассмотрения. Теперь уравнение (5) принимает вид

$$
\left(\bar{L}+C_{0} M\right) \alpha^{+}+C_{4} \alpha^{+} \xi \xi^{+}+C_{5} \alpha \xi^{+} \xi^{+}+C_{6} \alpha \alpha^{+} \alpha^{+}+C_{7} \xi \xi^{+} \xi^{+}=0,
$$

где $\bar{L}=C_{1} \partial_{t}+C_{2} \Delta / 2 m+C_{3}$. Числовые коэффициенты $C_{i}$ можно вычислять с необходимой точностью с помощью предложенной теории возмущений, однако, как мы увидим в разделе 7, их значения не важны при описании критического поведения. Также впоследствии окажется, что вклады с коэффициентами $C_{4}$ и $C_{5}$ дадут целиком ИК-несущественные вклады.

Следует использовать построенную нами теорию возмущений, чтобы получить локальную форму для динамического уравнения на поле плотности (10). Варьируя $-\Gamma_{\alpha}(11)$ и $-\Gamma_{\alpha^{+}}$и получив пропагаторы, подставим их в диаграммы (10). Разложим диаграммы в ряды по внешнему импульсу и частоте аналогично тому, как это было 
сделано для уравнения (5), и удержим лишь ИК-существенные вклады: $\omega M, \mathbf{k}^{2} M$, $\mathbf{k}^{2} \alpha \alpha^{+}, \mathbf{k}^{2} \xi \xi^{+}$. В результате получим

$$
\begin{aligned}
& B_{1} \partial_{t} M+B_{2} \Delta M+B_{3} \Delta\left(\alpha^{+} \alpha\right)+B_{4} \Delta\left(\xi^{+} \xi\right)+ \\
& \quad+B_{5}\left(\alpha^{+} \Delta \alpha-\alpha \Delta \alpha^{+}\right)+\xi^{+} \Delta \xi-\xi \Delta \xi^{+}=0 .
\end{aligned}
$$

Заметим, что вклад $B_{2} \Delta M$ генерируется благодаря зависимости от поля $M$ величины $\Gamma_{\alpha, \alpha^{+}}=\bar{L}+C_{3}+C_{0} M+C_{4} \xi \xi^{+}+2 C_{6} \alpha \alpha^{+}$и, следовательно, пропагатора $W_{A^{+} A}$ в диаграммах (10).

\section{6. СТОХАСТИКА ЖЕСТКИХ ПОЛЕЙ}

Итак, нам удалось записать уравнения стационарности Лежандра для полей $\alpha$, $\alpha^{+}$и $M$ в локальной форме. Чтобы привести их в соответствие со стандартной формой уравнений Ланжевена F-модели, будем ассоциировать последние слагаемые в (11) и (12) со случайной силой:

$$
f_{\alpha^{+}}=\lambda \xi \xi^{+} \xi^{+}, \quad f_{\alpha}=\lambda \xi \xi \xi^{+}, \quad f_{M}=\xi^{+} \Delta \xi-\xi \Delta \xi^{+}
$$

При этом оставшееся усреднение по жестким полям $\langle\cdot\rangle_{\xi}$ нам необходимо заменить на усреднение по введенным случайным силам.

Заметим, что, в отличие от обычных случайных сил уравнения Ланжевена, функция распределения введенных сил, очевидно, не гауссова. Например, для $f_{\alpha^{+}}$это распределение имеет вид

$$
\rho\left(f_{\alpha^{+}}\right)=\int D \xi D \xi^{+} \delta\left(f_{\alpha}-\lambda \xi \xi^{+} \xi^{+}\right) e^{-S\left(\xi, \xi^{+}\right)}
$$

что, в отличие от гауссова распределения, стремится к бесконечности при малых $f_{\alpha}$. Аналогично можно предъявить выражения и для распределений случайных сил $\rho\left(f_{\alpha}\right)$ и $\rho\left(f_{M}\right)$.

Провести усреднение по жестким полям удается, если воспользоваться в уравнениях (11), (12) диаграммной техникой Уальда. Обозначим линией со стрелкой пропагатор, соответствующий линейному оператору $\bar{L}$, зигзагом - жесткое поле $\xi$, а волнистой линией - коррелятор жесткого поля $D \equiv\langle\xi \xi\rangle_{\xi}$ (расстановка крестов на линиях по-прежнему призвана различать поля $\xi$ и $\xi^{+}$). Решим итерациями уравнения $-\Gamma_{\alpha}=-\Gamma_{\alpha^{+}}=0$, записанные в форме (11), относительно $\alpha, \alpha^{+}$, строя ряды относительно нелинейных вкладов по полям; поле плотности будем воспринимать согласно (9) как нелинейность по полям $\alpha, \alpha^{+}, \xi, \xi^{+}$. Решение будет иметь вид древесных диаграмм

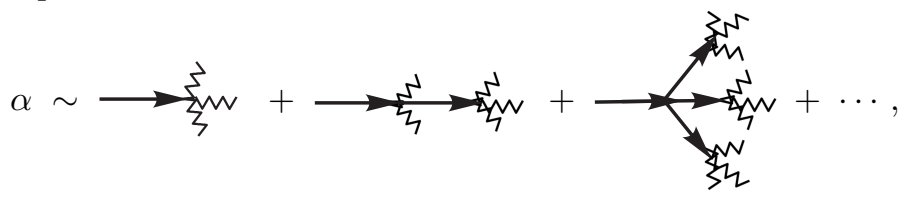


коэффициенты при диаграммах и расстановка крестов не указаны. Приведем некоторые из диаграмм, появляющихся при вычислении $\left\langle\alpha^{+} \alpha\right\rangle_{\xi}$ :

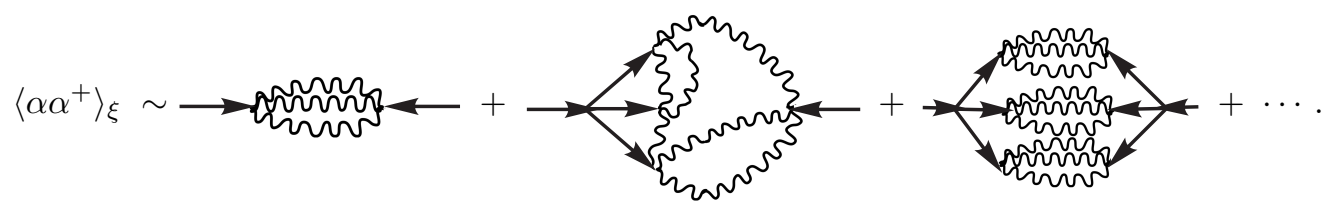

Покажем, что такое усреднение в любой диаграмме сводится в ИК-области к усреднению с пропагатором случайной силы:

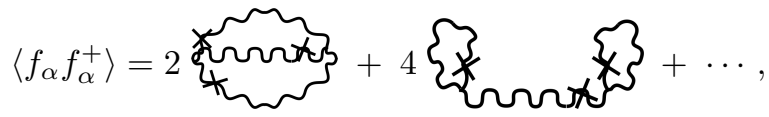

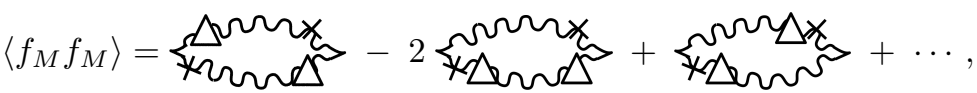

и что все остальные вклады в $\left\langle\alpha \alpha^{+}\right\rangle_{\xi}$ ИК-несущественны.

Рассмотрим, например, вторую из диаграмм в выражении для $\left\langle\alpha \alpha^{+}\right\rangle_{\xi}$. В ИК-области подграф, образованный волнистыми линиями, может быть разложен в ряды по внешним относительно подграфа импульсам и частотам $\omega_{i}, \mathbf{k}_{i}, i=1,2,3$. Первый член этого разложения $\left(\omega_{i}=\mathbf{k}_{i}=0\right)$ является наиболее ИК-существенным и соответствует исходному графу со стянутым в точку подграфом:

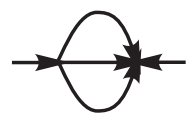

Однако в рамках MSR-формализма [9] подобный граф соответствует члену действия, пропорциональному $\left(\alpha^{\prime}\right)^{4}$ ( $\alpha^{\prime}$ - динамическая полевая переменная MSR-формализма, дуальная к полю $\alpha$ ). Соответствующая вершина, как хорошо известно из размерного анализа MSR-действия, является ИК-несущественной.

Несложно понять, что такие же рассуждения верны для всех диаграмм $\left\langle\alpha \alpha^{+}\right\rangle_{\xi}$, кроме тех, которые входят в пропагаторы случайных сил (и которые, в свою очередь, соответствуют членам $\left(\alpha^{\prime}\right)^{2}$ MSR-действия). По этой же причине все диаграммы, порождаемые членами с коэффициентами $C_{4}$ и $C_{5}$ в $(11)$, которые физически, очевидно, соответствуют мультипликативному шуму, следует отбросить как ИК-несущественные.

Наконец, в полученных выражениях для корреляторов случайных сил в ИК-области можно считать равными нулю все внешние импульсы и частоты. При этом, например, пропадает вторая диаграмма в выражении для $\left\langle f_{\alpha} f_{\alpha}^{+}\right\rangle$. Это означает, что в критической области функция распределения случайных сил может рассматриваться как белый шум.

\section{7. СООТВЕТСТВИЕ РЕЗУЛЬТАТОВ И Ғ-МОДЕЛИ}

Действию (1) соответствует в гиббсовском статическом пределе модель с функцией распределения $e^{-S_{\mathrm{st}}}$, где

$$
S_{\mathrm{st}}=\beta \psi^{+}\left(-\frac{\Delta}{2 m}-\mu\right) \psi+\frac{\beta \lambda}{2}\left(\psi^{+} \psi\right)^{2} .
$$


Полученные нами динамические уравнения для мягких полей и поля плотности имеют вид уравнений Ланжевена

$$
\partial_{t} \Phi=\Gamma \frac{\delta S}{\delta \Phi}+f, \quad \Phi \equiv\left\{\alpha, \alpha^{+}, M\right\}
$$

однако $S$ отличается от $S_{\mathrm{st}}$ традиционной F-модели множителями при членах действия. Отличие связано с нетривиальными коэффициентами $C_{i}$. Кроме того, уравнение Фоккера-Планка, соответствующее стохастическому уравнению (14), для функции распределения $P(\Phi, t)$

$$
\partial_{t} P+\frac{\delta}{\delta \Phi}\left(\frac{D}{2} \frac{\delta}{\delta \Phi}-\Gamma \frac{\delta S}{\delta \Phi}\right) P(\Phi, t)=0
$$

имеет не зависящее от времени решение с "правильным" статическим действием $P_{\text {st }}=e^{-S}$ лишь при "правильных" значениях коррелятора, $D=2 \Gamma$, что в нашем случае не так.

Тем не менее противоречия в рассмотрении гиббсовского статического предела данной модели нет. При $D \neq 2 \Gamma$ в статическом решении уравнения Фоккера-Планка действие лишь масштабируется: $P_{\mathrm{st}}=e^{-\Gamma S / D}$. Однако масштабирование этих членов не влияет на критическое поведение системы, которое полностью задается значениями инвариантных зарядов, определяемых методом ренормгруппы.

Таким образом, полученные стохастические уравнения Ланжевена в ИК-области соответствуют F-модели критической динамики.

Благодарности. Работа поддержана РФФИ (грант № 12-02-00874-a), Slovak Academic Information Agency (2011), Ministry of Education, Science, Research and Sport of Slovak Republic (грант VEGA 1/0222/13) и Center of Excellence for Nanofluid of IEP SAS.

\section{Список литературы}

[1] P. C. Hohenberg, B. I. Halperin, Rev. Mod. Phys., 49:3 (1977), 435-479.

[2] C. De Dominicis, L. Peliti, Phys. Rev. B, 18:1 (1978), 353-376.

[3] А.Н. Васильев, Функииональные методы в квантовой теории поля и статистике, ЛГУ, Л., 1976.

[4] М. В. Комарова, Д. М. Краснов, М. Ю. Налимов, ТМФ, 169:1 (2011), 89-99.

[5] А. А. Абрикосов, Л. П. Горьков, И. Е. Дзялошинский, Методы квантовой теории поля в статистической физике, Физматгиз, М., 1998.

[6] Л. В. Келдыш, ЖЖЭТФ, $47: 4$ (1964), 1515-1527.

[7] А. Н. Васильев, Квантовополевая ренормгруппа в теории критического поведения и стохастической динамике, Изд-во ПИЯФ, СПб., 1998.

[8] Ш. Ма, Современная теория критических явлений, Мир, М., 1980.

[9] P. C. Martin, E. D. Siggia, H. A. Rose, Phys. Rev. A, 8:1 (1973), 423-437. 